Prepared for the National Nuclear Security Administration,

Office of Defense Nuclear Nonproliferation and

Office of Nonproliferation Research and Development

under U.S. Department of Energy Contract DE-AC05-76RL01830

\title{
Measurement Protocols for Optimized Fuel Assembly Tags
}

\author{
DC Gerlach \\ CJ Gesh \\ MR Mitchell \\ DE Hurley \\ BD Reid
}

November 2008

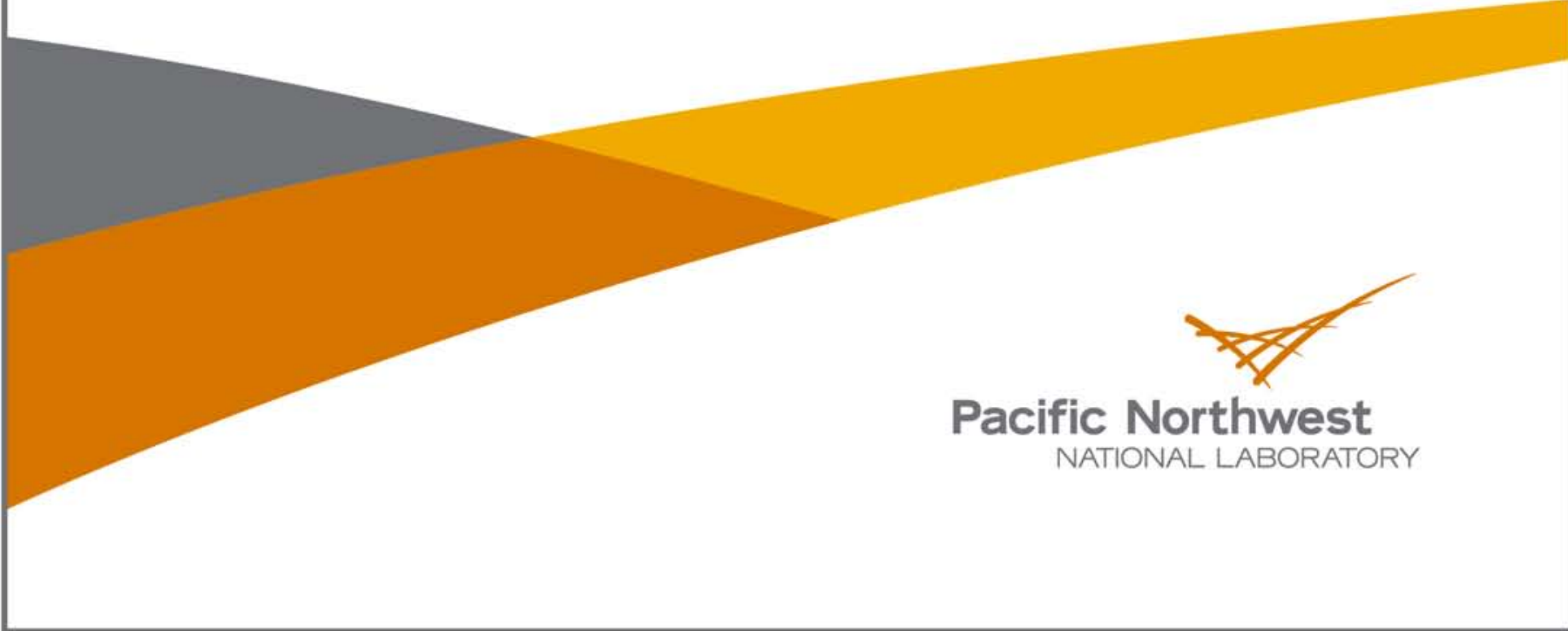




\title{
DISCLAIMER
}

This report was prepared as an account of work sponsored by an agency of the United States Government. Neither the United States Government nor any agency thereof, nor Battelle Memorial Institute, nor any of their employees, makes any warranty, express or implied, or assumes any legal liability or responsibility for the accuracy, completeness, or usefulness of any information, apparatus, product, or process disclosed, or represents that its use would not infringe privately owned rights. Reference herein to any specific commercial product, process, or service by trade name, trademark, manufacturer, or otherwise does not necessarily constitute or imply its endorsement, recommendation, or favoring by the United States Government or any agency thereof, or Battelle Memorial Institute. The views and opinions of authors expressed herein do not necessarily state or reflect those of the United States Government or any agency thereof.

\author{
PACIFIC NORTHWEST NATIONAL LABORATORY \\ operated by \\ BATTELLE \\ for the \\ UNITED STATES DEPARTMENT OF ENERGY \\ under Contract DE-AC05-76RL01830
}

Printed in the United States of America

Available to DOE and DOE contractors from the

Office of Scientific and Technical Information,

P.O. Box 62, Oak Ridge, TN 37831-0062;

ph: (865) 576-8401

fax: $(865) 576-5728$

email: reports@adonis.osti.gov

\footnotetext{
Available to the public from the National Technical Information Service, U.S. Department of Commerce, 5285 Port Royal Rd., Springfield, VA 22161 ph: (800) 553-6847 fax: $(703) 605-6900$

email: orders@ntis.fedworld.gov

online ordering: http://www.ntis.gov/ordering.htm
}

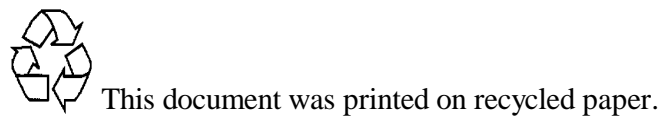




\title{
Measurement Protocols for Optimized Fuel Assembly Tags
}

\author{
DC Gerlach \\ CJ Gesh \\ MR Mitchell \\ DE Hurley \\ BD Reid
}

November 2008

Prepared for the National Nuclear Security Administration, Office of Defense Nuclear Nonproliferation and Office of Nonproliferation Research and Development under U.S. Department of Energy Contract DE-AC05-76RL01830

Pacific Northwest National Laboratory

Richland, Washington 99352 



\section{Summary}

In support of our ongoing signatures project, we present information on measurement protocols selected for possible use in optimized tags that could be applied to fuel assemblies to provide an objective measure of burnup. The amount and isotopic composition of plutonium produced in a fuel assembly can be calculated based on assembly burnup.

Important advantages of optimized tags are

- the small sample size afforded by the pure tag material as opposed to a Zircaloy sample, in which most of the sample does not provide any signal

- the absence of isotopes that activate, such as ${ }^{59} \mathrm{Co}$

- the absence of species that create isobaric interference with the isotope pairs of interest.

The advantages described above can either provide for a shorter "read" time in a laboratory-based secondary ionization mass spectrometry (SIMS) machine or allow analysis to be done in a miniature SIMS machine on the reactor site. 



\section{Acronyms and Abbreviations}

$\begin{array}{ll}\text { at\% } & \text { atom percent } \\ \text { BWR } & \text { boiling water reactor } \\ \text { Co } & \text { cobalt } \\ \text { cps } & \text { cycles per second } \\ \mathrm{Fe} & \text { iron } \\ \mathrm{H} & \text { hydrogen } \\ \mathrm{Hf} & \text { hafnium } \\ \mathrm{Ir} & \text { iridium } \\ \mathrm{IRM} & \text { isotope ratio method } \\ \mathrm{Ni} & \text { nickel } \\ \mathrm{O} & \text { oxygen } \\ \mathrm{Os} & \text { osmium } \\ \mathrm{PNNL} & \text { Pacific Northwest National Laboratory } \\ \mathrm{Pt} & \text { platinum } \\ \mathrm{Pu} & \text { plutonium } \\ \mathrm{SIMS} & \text { secondary ionization mass spectrometry } \\ \mathrm{Ti} & \text { titanium } \\ \mathrm{Zr} & \text { zirconium }\end{array}$





\section{Contents}

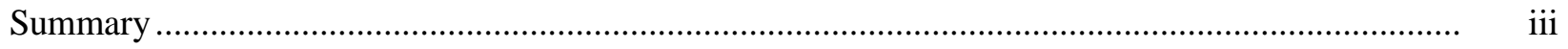

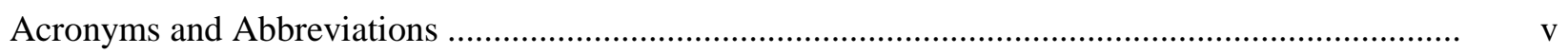

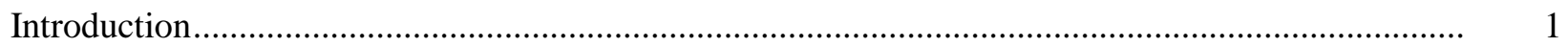

Secondary Ionization Mass Spectrometry (SIMS) Measurement Protocols for Optimized

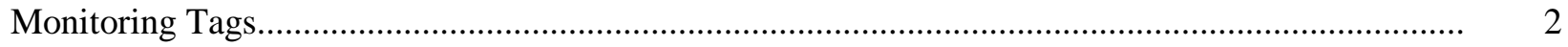

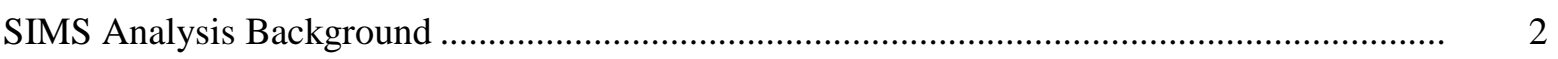

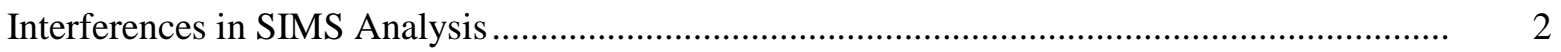

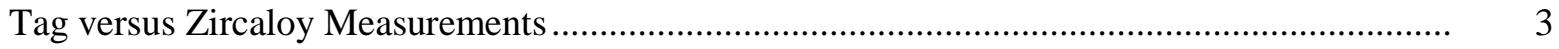

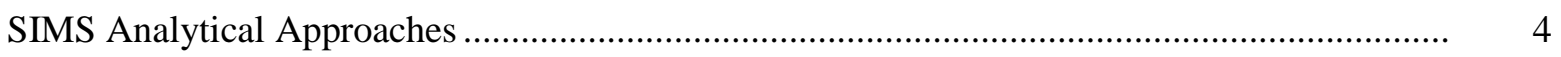

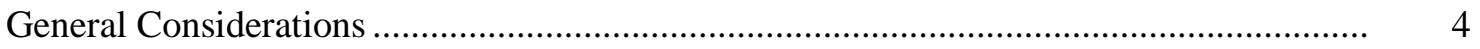

Specific Recommended Analytical SIMS Protocols for Optimized Tags........................... 4

Preferred Tag Elements/Materials ................................................................................... 5

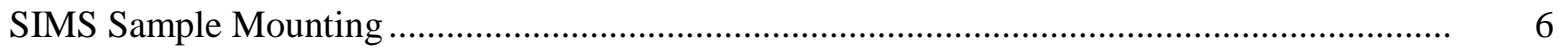

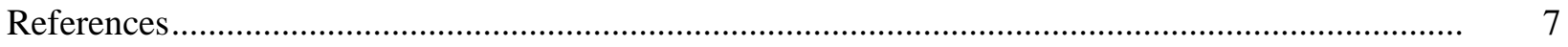

\section{Figure}

1 SIMS Mass Scan from Hydrogen to >Plutonium Mass Range ................................................. 3 



\section{Introduction}

This report describes the measurement protocols for optimized tags that can be applied to standard fuel assemblies used in light water reactors. The work was performed at Pacific Northwest National Laboratory for National Nuclear Security Administration Office of Nonproliferation Research and Development as part of research to identify specific signatures that can be developed to support counterproliferation technologies.

The isotope ratio method (IRM) estimates the energy production in a fission reactor by measuring isotope ratios in non-fuel reactor components. The isotope ratios in these components then can be related directly to the cumulative energy production with standard reactor calculations. Isotope ratio methods have been used to estimate total fluence at research reactors by measuring impurities in aluminum core supports (Cliff et al. 2005) and by measuring impurities in graphite from graphite-moderated reactors (Reid et al. 2001; Gesh 2004). One technique for measuring the relative abundance of two isotopes is the secondary ionization mass spectrometer (SIMS). It is particularly well adapted for atomic masses around 50 (Gerlach et al. 2006). Gerlach et al. (2006) discussed using impurities in the Zircaloy components of fuel assemblies, including measurements of samples taken from the fuel assembly channels of commercial boiling water reactors (BWRs) (Gerlach et al. 2007).

Rather than relying on trace impurities, the optimized tag approach involves attaching a small tag made of specific isotopes that have desirable nuclear, chemical, and ionization characteristics. One necessary nuclear characteristic is having at least two stable isotopes for isotope ratio measurement. Optimal isotopes have a neutron capture cross section low enough that they have a meaningful variation over anticipated fuel assembly irradiation, have good corrosion resistance and chemical compatibility with the fuel assembly Zircaloy and coolant, and have atomic weights significantly higher or lower than major interference species in the SIMS machine.

Given enough time and a laboratory-sized SIMS instrument, measurements on trace element isotope ratios in Zircaloy structural components removed from the assembly can provide useful verification information. However, the optimized tags should enable shorter measurement times and/or the use of portable SIMS instruments for analysis at the reactor site. 


\section{Secondary lonization Mass Spectrometry (SIMS) Measurement Protocols for Optimized Monitoring Tags}

\section{SIMS Analysis Background}

Secondary ionization mass spectrometry analysis is a well-established analytical method that is conducted directly on samples with little or no preparation or chemical separation. It offers advantages of quick turnaround compared to that offered by other mass spectrometric analysis methods. Secondary ionization mass spectrometry uses a beam of high-energy primary ions to sputter secondary ions from the sample surface and is capable of high spatial resolution.

Secondary ionization mass spectrometry is capable of microscopic physical spatial resolution in addition to elemental or isotopic analysis. This capability is due to both the spatial resolution afforded by using a primary ion beam for initial sputtering and the secondary ion optics. Some ion beams can sample small areas down to 1 micron in diameter, a much smaller spot size than achievable by laser ablation. Spatial resolution provided by SIMS allows analysis of microscopic features in a sample material or microscopic objects and evaluation of microscopic elemental and isotopic heterogeneity in a very small area. Thus, the overall size of the monitoring tags can be very small (a few square millimeters).

The detection efficiency for SIMS is comparable to other mass spectrometers. However, this efficiency may vary with the isotope being analyzed and the composition of the sample matrix. The metallic elements selected for optimized monitoring tags were chosen specifically for rapid and accurate SIMS analyses. The amount of sample consumed by the SIMS primary ion beam is generally very small, less than a few cubic microns. As in other mass spectrometers, molecular and isobaric interferences must be avoided or compensated for during SIMS analysis.

\section{Interferences in SIMS Analysis}

Mass spectrometric measurements, especially those conducted on unprocessed solid samples or sample solutions, include molecular or other types of ions which may have the same molecular mass as the isotope of interest resulting in potential interferences. Many of these can be accommodated by making corrections or using strategic instrument settings. When interferences must be avoided, laborintensive chemical separation and isolation of the element of interest from the sample matrix must be performed. In our case, direct analysis of solid samples is necessary; interferences must be avoided or removed, or measures taken to correct analytical results for interferences.

Interferences in SIMS analysis can include combinations of matrix ions (M), trace impurity ions (T) with oxygen (O) from the primary ion beam or with trace gases (e.g., hydrogen) in the high-vacuum sample chamber or arising from the sample material itself. In addition to isobaric interferences such as ${ }^{50} \mathrm{Cr}^{+}$and ${ }^{50} \mathrm{Ti}^{+}$, interferences in any material undergoing SIMS analysis will include $\mathrm{M}_{\mathrm{a}} \mathrm{M}_{\mathrm{b}}{ }^{+}(\mathrm{a}, \mathrm{b}=$ different matrix elements), $\mathrm{M}_{\mathrm{n}}^{+}\left(\mathrm{n}=2,3\right.$, etc.), $\mathrm{MO}^{+}, \mathrm{MO}_{\mathrm{n}}^{+}\left(\mathrm{n}=2,3\right.$, etc), $\mathrm{M}^{+2}, \mathrm{M}^{+3}, \mathrm{MT}^{+}, \mathrm{TO}^{+}$, and $\mathrm{TH}^{+}$, in decreasing order of probability. However, some specific interference effects remain significant, regardless of magnitude, if they inhibit or prevent desired measurements. This was the case for the Zircaloy samples analyzed for proof-of-principle demonstrations. Because zirconium isotopes do not vary strongly with fluence, and because the Zircaloy samples also included several other matrix or 
alloying elements, choices of trace impurity elements as indicators of fluence were restricted. Some examples are shown in Figure 1.

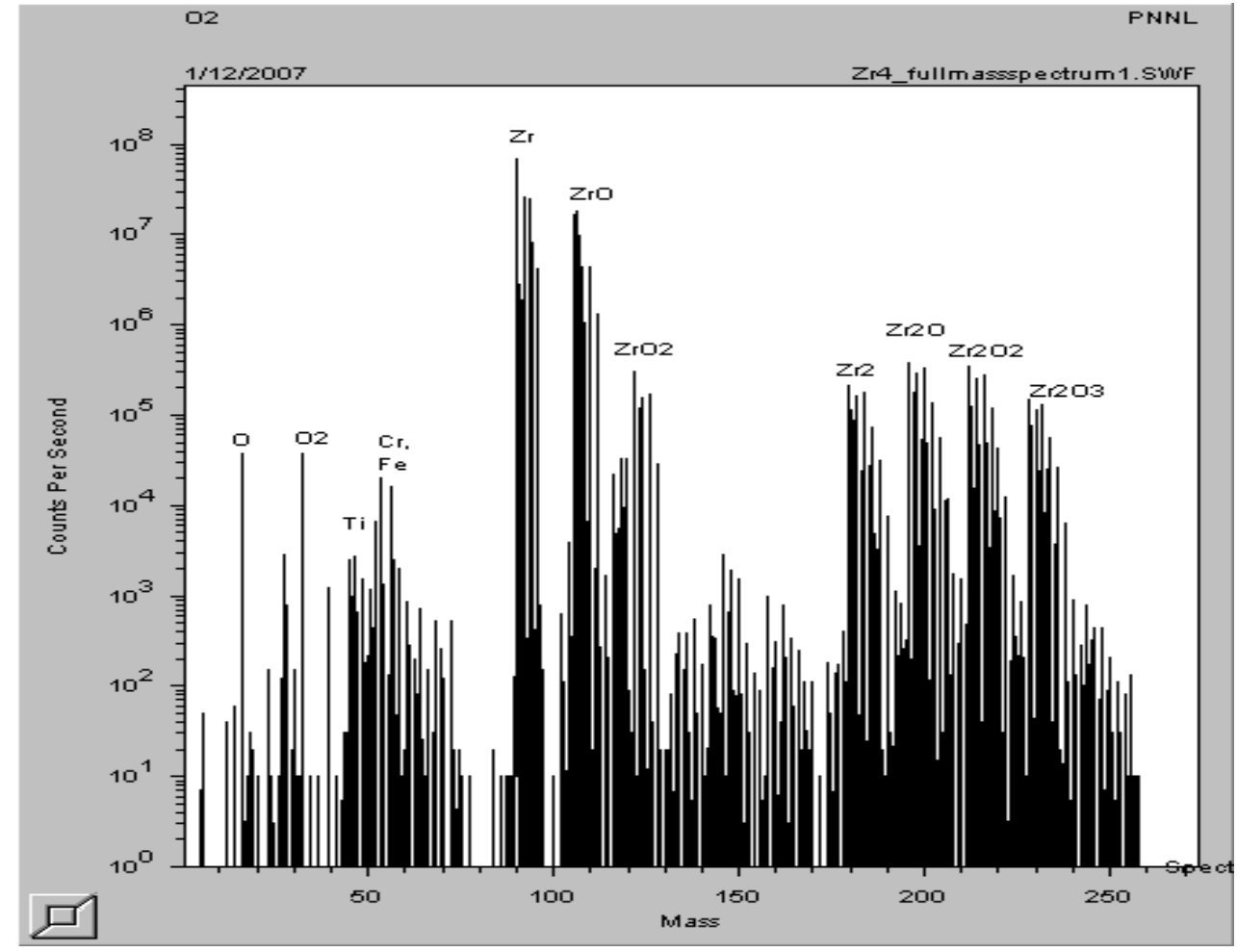

Figure 1. SIMS Mass Scan from Hydrogen to >Plutonium Mass Range. Matrix elements (zirconium) and major interferences arising from zirconium and the SIMS primary oxygen ion beam are shown.

\section{Tag versus Zircaloy Measurements}

Many of the complex analytical strategies and protocols used for trace-element zircaloy sample analysis can be largely avoided by using high-purity non-alloyed single-element materials. For titanium isotope ratios in Zircaloy, it was necessary to monitor doubly charged $\mathrm{Zr}^{+2}$ interferences that occurred from ${ }^{46} \mathrm{Ti}$ to ${ }^{48} \mathrm{Ti}$. This was possible only because of the existence of an odd-numbered isotope such as ${ }^{93} \mathrm{Zr}$, which could be monitored at a half-mass isotope position, as described in previous reports (Gerlach et al. 2007). The appropriate amounts of ${ }^{96} \mathrm{Zr}^{+}$ion counts were then subtracted to obtain a corrected amount of ${ }^{48} \mathrm{Ti}^{+}$ion counts for ${ }^{49} \mathrm{Ti} /{ }^{48} \mathrm{Ti}$ ratio measurements. For hafnium isotope ratios, $\mathrm{M}_{3}{ }^{+}(\mathrm{M}=\mathrm{Fe}, \mathrm{Ni})$ molecular interferences occurred at all hafnium isotopes. Because isotopes with lower mass generally have less scattering and less energy width compared to heavier isotopes in magnetic sector mass spectrometry, it was possible to accurately measure hafnium secondary ions by using a combination of different physical and electronic settings to occlude the more narrow $\mathrm{M}_{3}{ }^{+}$interferences. 


\section{SIMS Analytical Approaches}

\section{General Considerations}

SIMS analysis is optimal for flat surfaces, and tag material lots must be chosen to provide at least a few square millimeters of surface area for analysis. Doing so will also provide enough area to avoid microscopic defects or impurity-rich inclusions in the tag material that could bias measurements. Optimal areas for analysis should be chosen using SIMS ion imaging approaches, after a few minutes of presputtering a chosen area with the SIMS primary ion beam to remove thin films or surface contamination.

Some specific approaches and instrument settings for analysis of graphite samples are described in Battelle Procedure 320-65-01, Rev. 1, "Secondary Ionization Mass Spectrometry Analysis of Graphite and Associated Samples.” These serve as examples of approaches to be taken in analysis of tag materials. In general, it is desirable to obtain a high ion count rate (up to 500 thousand cps) of the most abundant isotope to be measured because much of the measurement uncertainty is inversely proportional to the square root of the count rate. Instrument or detector backgrounds must be considered, but the effective dynamic range for ion-counting ratio measurements in SIMS can be five orders of magnitude using only the ion-counting detector. This should suffice for measurement of burnup ratios in the chosen tag materials, even if one isotope is completely consumed and undetectable above instrument background.

A variety of instrument settings can be adjusted to obtain optimal ion count rates, but the most significant are adjustments in the density and strength of the primary ion beam bombarding the sample material. For example, to obtain ${ }^{49} \mathrm{Ti}$ or ${ }^{178} \mathrm{Hf}$ ion count rates of even $1000 \mathrm{cps}$ in the Zircaloy samples, it was necessary to use a primary ion beam of 1 to 2 microamperes, the resulting zirconium matrix ion count rates were 10 million to 50 million cps. If the tag materials consist of matrix elements as target elements, rather than impurity elements at the parts-per-million level as measured in the Zircaloy samples, a much lower primary ion beam of a few nanoamperes can be used, consuming less sample. In this case, any trace elements and their interference effects on ratio measurements for the matrix element will be negligible and several orders of magnitude lower compared to ion count rates for matrix elements.

\section{Specific Recommended Analytical SIMS Protocols for Optimized Tags}

The PNNL work led to six specific recommendations:

1. Obtain two or more lots of the selected high-purity material to support testing. Keep some in reserve to represent initial non-irradiated reference material.

2. For both non-irradiated material and irradiated tags, mount a portion of the material in the standard SIMS sample holder and pre-sputter area for analysis using a primary ion beam of approximately 1-2 microamperes. This is necessary because surface impurities can be added or embedded when high-purity metals are formed or rolled into thin foils or filaments.

3. Conduct an elemental scan from hydrogen through plutonium or higher, with the high primary ion beam, to identify impurity elements and potential isobaric and molecular interferences. Conduct the element scan using the lower primary ion beam, with matrix element isotopes at approximately 500 thousand cps, to again assess impurity elements and interferences These interferences should be negligible, with the exception of $\mathrm{MO}+, \mathrm{MOn}^{+}$, or $\mathrm{Mn}^{+}$. Switch to the ion-imaging mode and, using the lower-density primary ion beam, conduct a search for possible impurity-rich microscopic 
domains or inclusions in each chosen analysis spot. If present, select another spot for pre-sputtering and analysis.

4. In setting up a data acquisition routine, select individual isotope counting times to optimize measurement precision. In general, longer counting times are required for low-abundance isotopes or for isotopes predicted to be low in abundance after burnup, relative to isotopes with lower cross sections.

5. In non-irradiated samples held in reserve as reference material, conduct replicate analyses to determine the optimum length of time for individual spot analyses and the optimum number of spot analyses required to minimize measurement uncertainty. Conducting these measurements will provide the initial starting values to use in neutronics modeling and plutonium production estimates. By measuring the reserved material from the same lot from which tag materials were produced, SIMS instrument measurement mass bias, which can be up to several percent relative to certified values, can be disregarded. Long-term changes in measurement results on the non-irradiated reference material are of interest mainly for instrument maintenance and checking the ion-counting detection system on the SIMS.

6. Analyses of non-irradiated reserved reference samples should be conducted along with analyses of irradiated tags. Long-term instrument drift may result in small changes in the measurement mass bias, and it is best to use recent or interleaved measurements to determine initial ratio values for calculations. The same analysis menu and isotope counting times should be used for both nonirradiated reference samples and irradiated tags. Results for both tags and non-irradiated starting material should be reported.

\section{Preferred Tag Elements/Materials}

As discussed elsewhere, preferred elemental tag materials include titanium, hafnium, and platinum based on cross sections of their various isotopes, corrosion resistance, and predicted activation products. These elements are available in refined purity grades in foil or flattened ribbon forms, ideal for SIMS analyses. The cost of tags using high-purity grades will not be prohibitive because only small portions are needed to make the tags — one of the advantages of SIMS analysis and utilization of small sample areas. Using smaller portions in tags also will reduce the amount of radioactivity exposure during sample handling, shipment, and SIMS analysis.

Titanium can be obtained in very high purity up to 99.9999\%; however, 99.99\% should be sufficient. The most common impurities found in titanium include iron, hafnium, $\mathrm{Nb}$, zirconium, or tantalum, and none of these impurity elements would create any $\mathrm{MO}^{+}$or $\mathrm{M}^{+\mathrm{n}}$ interferences that could affect measurement of ${ }^{49} \mathrm{Ti} /{ }^{48} \mathrm{Ti}$ ratios. The other titanium isotopes change much less with fluence exposure but may be worth monitoring in high-burnup samples.

Titanium is available also as an alloy with hafnium or with hafnium at an impurity level of $>10 \mathrm{ppm}$. This option obviously provides two indicator elements as well as increased versatility and crosschecks. If the alloy is certified as $99.99 \%$ or higher purity, then the effects of $\mathrm{M}_{3}^{+}(\mathrm{M}=\mathrm{Fe}, \mathrm{Ni})$ interferences from iron and nickel impurities should be negligible on hafnium isotope ratio measurements. An advantage of using high-purity hafnium with titanium as an impurity is that the minor isotope of ${ }^{174} \mathrm{Hf}(0.16$ at\%) may provide an additional indicator. Lower levels of ${ }^{177} \mathrm{Hf}$, which has a very high cross section, may be measurable to higher burnup levels than as an impurity element in the Zircaloy, at least until ${ }^{177} \mathrm{Hf}$ is at 
instrument background. Finally, ${ }^{180} \mathrm{Hf}$ will be usable as an indicator isotope, either as an impurity in titanium or as a matrix element. Earlier in Zircaloy samples, ${ }^{90} \mathrm{Zr}_{2}{ }^{+}$interferences prevented measurement of ${ }^{180} \mathrm{Hf}$.

Platinum also is available in very high-purity grades, in foils or flattened ribbons, and is very robust and resistant to corrosion in extreme settings. Expected impurities in platinum include iridium and osmium, which also are possible candidate tag elements. However, osmium has poor corrosion resistance. As an impurity element in platinum, ${ }^{184}$ Os to ${ }^{189}$ Os may be useful if present to several parts per million or more, whereas ${ }^{190}$ Os and ${ }^{192}$ Os have isobaric interferences with ${ }^{190} \mathrm{Pt}$ and ${ }^{192} \mathrm{Pt}$. For iridium to be useful as an impurity element in platinum, iridium may need to be present as a higher-level alloying element at $1000 \mathrm{ppm}$ or higher to overcome ${ }^{190} \mathrm{PtH}^{+}$and ${ }^{192} \mathrm{PtH}^{+}$interferences on ${ }^{191} \mathrm{Ir}$ and ${ }^{193} \mathrm{Ir}$, the only two isotopes of iridium.

\section{SIMS Sample Mounting}

Commercially available sample mounts made for the SIMS instrument provide a wide range of options for sample mounting. For small foils or portions of ribbons, it may be advantageous to design an insert that can be quickly mounted in a standard SIMS sample holder. The insert would be designed in a manner to allow quick mounting of a small sample foil or clipped ribbon portion, and could be inexpensive and disposable, so that the more expensive SIMS sample holders can be re-used many times over. Mounting of sample material in the insert should avoid use of adhesives, since a conductive contact must be established and maintained in the SIMS sample holder. Newly available conductive adhesives should not be considered, since they may contribute background and trace amounts of gases in the high vacuum SIMS sample chamber. 


\section{References}

Cliff JB, DP Frank, GJ Gesh, WW Little, Jr., GH Meriwether, RT Pagh, BD Reid, CW Becker, PA Simpson, and RE Touchberry. 2005. "Isotope Ratio Method Analysis of the Ford Nuclear Reactor." Presented to the Joint Meeting of the National Organization of Test, Research, and Training Reactors and the International Group on Research Reactors, September 12-16, 2005, Gaithersburg, Maryland. Available at http://www.ncnr.nist.gov/trtr2005/Proceedings/Gesh\%20\%20Isotope\%20Ratio\%20Method\%20FNR.pdf (November 2008).

Gerlach DC, JB Cliff, DE Hurley, BD Reid, WW Little, GH Meriwether, AJ Wickham, and TA Simmons. 2006. "Secondary ionization mass spectrometric analysis of impurity element isotope ratios in nuclear reactor materials.” Journal of Surface Science 252:7041-7044.

Gerlach DC, BD Reid, MR Mitchell, GJ Gesh, and DE Hurley. 2007. Determination of Light Water Reactor Fuel Burnup with the Isotope Ratio Method. PNNL-17053, Pacific Northwest National Laboratory, Richland, Washington.

Gesh CJ. 2004. A Graphite Isotope Ratio Method Primer: A Method for Estimating Plutonium Production in Graphite Moderated Reactors. PNNL-14568, Pacific Northwest National Laboratory, Richland, Washington.

Reid BD, DC Gerlach, and PG Heasler. 2001. Trawsfynydd Plutonium Estimate. PNNL-13528, Pacific Northwest National Laboratory, Richland, Washington. 




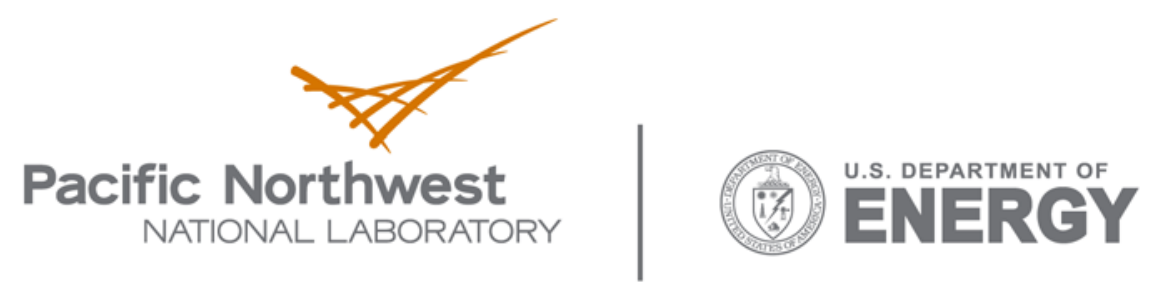

902 Battelle Boulevard

P.O. Box 999

Richland, WA 99352

1-888-375-PNNL (7665)

www.pnl.gov 